\title{
La figura ed i compiti processuali del Vescovo diocesano nel processo matrimoniale secondo la riforma del Papa Francesco
}

Since the beginning of the pontificate of Pope Francis we have heard in his preaching the calling to reform the structures of the church. Bergoglio presented his pastoral-evangelical idea of the functioning of the Church in the exhortation Evangelii Gaudium. Naturally the reform made by the Bishop of Rome also applies to the canon law. Directed by voices of hierarchy gathered at the Synod of Bishops 2014 and the spiritual good of the faithful, who are waiting for an explanation of their canonical situation, 15 August 2015 Pope Francis promulgated the Motu proprio Mitis ludex Dominus lesus, to accelerate and simplify the process of annulment of the marriage. Pope wanted the heart of the faithful were not too long enslaved by darkness of doubt because of delays in the judgment. In the center of his reform

Pope put the diocesan Bishop, who together with the Bishop of Rome becomes the guarantor of the unity of the Catholic faith and discipline. According to the principles of the reform, the bishop not only organizes and is responsible for the court structure in the local church, but he also has to personally fulfill the task of the first judge of the faithful, for whom he was established a shepherd. The new laws allow the bishop to establish a single judge and introduces the institution of processus brevior, which is provided for particular obvious situations. The only judge in this process is the bishop. It seems that the reform process of marriage, made in the spirit of historical continuity, will bring much spiritual fruit and contribute to the perception of the person the diocesan bishop not only as pastor bonus, but at the same time as iustus iudex.

Key words: Pope Francis, the process of marriage, the diocesan Bishop, judge, nullity of marriage, processus brevior, reform. 


\section{Introduzione}

Una delle parole che potrebbero caratterizzare il ponitificato del Papa Francesco è proprio la riforma. Practicamente dall'inizio del suo ministero Papa Bergoglio chiama tutta la Chiesa alla riforma ${ }^{1}$. Lo spirito della riforma si sente molto nella sua prima esortazione apostolica Evangelii Gaudium dove il successore di Pietro ci lascia il programma della sua missione apostolica. Il Papa indica un principio di conversione delle strutture ecclesiastiche per creare ,la Chiesa povera per i poveri"'. Gaurdando il pontificato del Papa Bergoglio possiamo notare, che questa riforma si fa anche nell'ambito del diritto canonico. Ultimamente sentiamo sulla riforma della Curia Romana, che sta svolgendo $\mathrm{Papa}^{3}$, ma egli ha emanato anche alcuni Motu proprio per cui ha modificato alcuni canoni nel Codice di Diritto Canonico ${ }^{4}$. Il cambiamento più grande è collegato con i Motu proprio Mitis Iudex Dominus Iesus (per la riforma del processo di dichiarazione di nullità del matrimonio nel Codice di Diritto Canonico) e Mitis et misericors Iesus (per la riforma del prprocesso di nullità del matrimonio nel Codice dei Canoni delle Chiese Orientali) - tutte due dal 8 settembre 2015. Con la sua legislazione Papa Francesco risponde alle richieste dei tanti vescovi, che hanno partecipato alla III Assemblea Generale Straordinaria del Sinodo dei Vescovi - ,un grande numero dei Padri ha sottolineato la necessità di rendere più accessibli ed agili, possibilmente del tutto gratuite, le procedure per il riconoscimento dei casi di

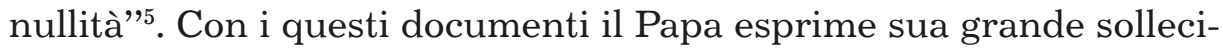
tudine e preocupazione della famiglia odierna. Il Papa Bergoglio non vuole, che il cuore dei fedeli che attendono il chiaramento del proprio stato non sia lungamente opresso dalle tenebre del dubbio ${ }^{6}$.

$1 \quad$ Per descrivere questa realtà il Santo Padre Francesco usa molto spesso altri termini ad esempio: conversione o rinnovamento ecclesiale.

2 Cf. Francesco, Udienza ai rappresentati dei media, Discorso del Santo Padre, Aula Paolo VI, 16 marzo 2013, http://w2.vatican.va/content/francesco/it/speeches/2013/march/documents/papa-francesco_20130316_rappresentanti-media. html. (10.01.2017).

3 Recentemente Papa Francesco ha stabilito i nuovi dicasteri: Dicasterio per il servizio dello sviluppo umano; Dicastero per i laici, la famiglia e la vita.

4 Ad esempio: la Lettera Apostolica in forma di Motu proprio del Sommo Pontefice Francesco: De concordia inter codices, emanata 31 maggio 2016.

5 III Asemblea Generale Straordinaria del Sinodo dei Vescovi, Relatio Synodi, Città del Vaticano 2014, 48.

$6 \quad$ Cf. Lettera Apostolica Mitis Iudex Dominus Iesus data Motu Proprio dal Santo PadreFrancesco sulla riforma del processo canonico per le cause di dichiarazione 
$\mathrm{Al}$ centro della sua riforma Papa Francesco pone la figura del Vescovo diocesano, che è costituito per la sua Chiesa locale pastore e capo, per ciò lui stesso è giudice tra i fedeli gli affidati. Francesco sapendo la delicatezza delle cause matrimoniali ci spiega, che il Vescovo diocesano assieme con il successore di Pietro, può essere il maggiore garante dell'unità cattolica nella fede e nella disciplina ${ }^{7}$. In questa ricerca cercheremo di indicare la centralità della figura del Vescovo diocesano nel rinnovamento del processo per la dichiarazione la nullità del matrimonio. Lo indicheremo come punto di riferimento per tutta la riforma voluta da Papa Francesco. Vorremo mostrare la figura del Vescovo diocesano come giudice del suo gregge, sopratutto in materia di questo processo e vorremo precisare i suoi compiti nell'ambito processuale previsti dalla nuova normativa canonica. Ovviamente, indicando la posizione ed i compiti processuali del Vescovo diocesano vogliamo scoprire lo spirito della riforma francescana.

\section{Il processo di dichiarazione di nullità del matrimonio nella storia della Chiesa}

Il processo matrimoniale ha la sua lunga storia. Per tanti secoli le cause di nullità matrimoniale erano giudicate secondo i principi generali del diritto processuale. Sempre il Vescovo come capo della Chiesa locale è conosciuto come primo giudice per i suoi fedeli. La storia della Chiesa dopo l'editto di Milano (313) conosce l'istituzione dell' episcopalis audientia - una forma di amministrazione della giustiza con valenza civile affidata ai Vescovi, l'atribuzione della potestà concerneva quasi tutte le possibli materie controverse (escluse quelle penali), non era limitata all'ambito religioso ${ }^{8}$.

Nel Medioevo la figura del Vescovo continua ad essere centrale nella organizzazione della giustizia ecclesiastica. Si creano le funzioni variamente denominate di arcidiaconi o decani, che in alcune materie amministrano in loco la giustizia. Le nuove figure dei giudici locali aiutano ai Vescovi compiere la loro potestà giudiziaria e guarantiscono la prossimità e la tempestività 9 . A partire dall XII secolo siamo testimoni di costituzione di un nuovo fenomeno: Officiale - è un spe-

di nullità del matrimonio nel Codice di Diritto Canonico (dopo: MIDI), Coletti a San Pietro 2015, p. 3.

7 Cf. MIDI, p. 3.

8 Cf. M. del Pozzo, Il processo matrimoniale più breve davanti al Vescovo, Roma 2016, p. 46. 
cifico ministro con la potestà giudiziaria deputato a esaminare in sua voce le cause ${ }^{10}$. L'officialità in genere è una forma di rappresentanza del Vescovo nell'esercito della giurisdizione, ovviamente distinta dal vicario generale. Come la consequenza di queste novità il Vescovo - il Pastore - si libera progressivamente di alcuni oneri ed incombenze. Nel Medioevo esiste un principio nullum divortium sine iudicio Ecclesiale, che suggellava la difesa dell'indissolubilità e il carattere propriamente spirituale del giudizio. La competenza vescovile in materia matrimoniale si ritenva esclusiva ma non assoluta ${ }^{11}$.

Quando il processo matrimoniale è diventato troppo complesso, si sentiva bisogno di semplificazione, rapidità ed abbreviazione nella giurisdizione. Lo vede Papa Clemente III e con la costituzione Saepe contingit dal 1306 permette di svolgere la procedura d'accertamento nel modo abbreviato. Per quello, secondo il prof. M. del Pozzo questo tipo di processo stabilito da Papa Celemente V può essere chiamato oggi come una sorta di antesignano per il processus brevior stabilito da Papa Francesco, di cui parleremo dopo ${ }^{12}$.

Il Concilio di Trento (1545-1563) ha riaffermato la dottrina sul matrimonio e ribadito l'esclusività della giurisdizione ecclesiastica nelle cause spirituali. Nell'ambito della disciplina processuale il Concilio di Trento stabilisce, che le cause di nullità del matrimonio devono essere giudicate dal Tribunale del Vescovo diocesano, ciò significa davanti al Vescovo diocesano, non invece dagli altri giudici inferiori che svolgevano loro servizio nel teritorio della diocesi (arcidiaconi o diaconi). Per questo si sottolineava l'incompetenza assoluta dei giudici inferiori. Si puntualizzava quindi il deferimento delle cause matrimoniali all'esame e alla giurisdizione del Vescovo. Le disposizioni del Concilio hanno rappresentato la falsariga dell'organizzazione giudiziaria ecclesiastica fino alla modernità. Il tribunale diocesano costituì il prototipo della giustizia locale. Per questo il Vescovo riacquistò così la diretta resposabilità della cause matrimoniali su base territoriale. Malgrado gli sforzi della riforma tridentina, taluni abusi sopratutto nella prassi della giustizia matrimoniale erano continuati ${ }^{13}$.

Il Papa Benedetto XIV vedendo alcuni abussi sopratutto nella prassi della giustizia matrimoniale, essendo un giurista, cercò con una serie di provvedimenti di dare nuovo vigore e rigore alla giurisdizione

10 Cf. C. Fantappiè, Storia del diritto canonico e delle istituzioni della Chiesa, Bologna 2011, p. 146-147.

11 Cf. M. del Pozzo, op. cit., p. 51.

12 Cf. M. del Pozzo, op. cit., p. 52.

13 Cf. Ibidem, p. 53-54. 
ecclesiastica sopratutto in materia matrimoniale. Con la Costituzione apostolica Dei miseratione, promulagata il 3 di novembre 1741, stabilisce l'ufficio del difensore del vincolo e l'esigenza della doppia sentenza conforme ${ }^{14}$. Nel pontificato del Papa Pio X vengono restituiti i tribunali della Santa Sede: la Rota Roma e la Segnatura Apostolica ${ }^{15}$.

La codifficazione del 1917 recepisce e stabilizza il modello delineato a Trento e gli sviluppi dettati dalla prudenza giuridica. Si parla del processo du nullità del matrimonio nei canoni 1960-1992 - la maggior parte recepite da Benedetto XIV. Nelle cause matrimoniali si ribadisce la cognizione del giudice del luogo in base ai titoli di competenza ${ }^{16}$. Si prevede anche tuttavia la competenza personale dell'Ordinario del luogo per le cause expceptae. La normativa codiciale viene riempita dopo dall'istruzione del Pio XI Provida Mater, che precisa le prescrizioni sulla formazione del tribunale collegiale e precisa l'inopportunità della presidenza del Vescovo. L'istruzione conferma peraltro l'esclusività della spettanza dell'Ordinario a dichiarare la nullità in processo documentale ${ }^{17}$.

Durante il Concilio Vaticano II (1962-1965) tanti vescovi facevano le richieste di riformare la procedura nelle cause matrimoniali, di cui si trattava dal 1963 nel dibatto della Pontificia Comissione per la revisione del Codice di Diritto Canonico ${ }^{18}$. Le domande della riforma del processo erano sentite anche durante il Sinodo dei Vescovi nel 1970 ed erano proproste dai tanti canonisti. Sopratutto si domandava della semplificazione della procedura nella seconda istanza ${ }^{19}$. Nel periodo postconciliare il Papa Paolo VI (ancora prima della nuova codificazione del 1983) ha promulgato il Motu proprio Causas matrimoniales, dove sottolineando il principio dell'indissolubilità ha stabilito alcune novità: un uomo laico può compiere l'ufficio del giudice nel collegio

14 Cf. Benedictus XIV, Constitutio Dei miseratione, [in:] Codicis Canonici fontes, I, P. Gasparri (a cura di), Romae 1923, p. 695-701.

15 Cf. Pius Pp. X, Constitutio apostolica Sapienti consilio, Romae 29.06.1908, http:// https://w2.vatican.va/content/pius-x/la/apost_constitutions/documents/ hf_p-x_apc_19080629_sapienti-consilio-index.html (4.11.2016).

16 Cf. M.del Pozzo, op. cit., p. 55-56.

17 Cf. W. Góralski, Wprowadzenie do motu proprio Mitis Iudex Dominus Iesus, [in:] Proces matżeński wedtug motu proprio Mitis Iudex Dominus Iesus, J. Krajczyński (a cura di), Płock 2015, p. 10-11.

18 Cf. Acte et documente Consilio Oecumenico Vaticano II apparando, series I, Appendicis vol. II, p. II, Romae 1961, p. 580-593.

19 Ad esempio I. Gordon, il professore del diritto processuale alla Pontificia Universitá Gregoriana nella sua opera dal 1969 De nimia preocessuum matrimonialium duratione ha prerarato alcune proposte e cambiamenti in tale ambiente. 
dei giudicanti, la possibilità in concreti casi - eccezionalmente - di nominare un giudice unico; amettere ai laici alcuni compiti: per esempio potrebbereo svolgere l'ufficio del notaio. ${ }^{20}$.

Nel Codice di Diritto Canonico promulagato da Giovanni Paolo II nel 1983 si parla del processo di dichiarazione di nullità del matrimonio nei canoni 1671-1691. La maggior parte dei canoni sono percepiti dalla costituzione apostolica di Paolo VI Causas matrimoniales. Le norme vengono riempite dalla Istruzione Dignitas connubii dal 25 gennaio $2005^{21}$. Il Codice di Diritto Canonico dal 1983 capisce il processo di dichiarazione di nullità del matrimonio come processo specciale, per cui si applica anche le norme del processo ordinario contezioso, le cause vengono tratte per la via giudiziale ${ }^{22}$. Ovviamente il CIC 1983 ribadisce la normativa stabilita da Papa Benedetto XIV di un obbligo della doppia conferma sentenza ${ }^{23}$. La legislazione non prevede nessuna speciale possibilità per il Vescovo per intervenire nel processo o nessuna forma abbraviata del processo, ecceto il processo documentale.

\section{I presupposti e principi della riforma del processo matrimoniale secondo Francesco}

Come avevamo detto la riforma del processo matrimoniale viene richiesta direttamente dai padri sinodali presenti nel Sinodo Straordinario nel 2014. Come afferma il prof. M. del Pozzo, la riforma fatta dal Papa Francesco deve essere riconosciuta come una determinazione voluta e richiesta dal basso. Lintervento risponde infatti tempestivamente e risolutamente alle reiterate sollecitazioni dei Vescovi in merito alla maggior celerità e snellimento dei giudizi canonici di nullità matrimoniale e al vivo interesse del Romano Pontefice per l'attegiamento ecclesiale nei confronti della pastorale familiare ${ }^{24}$. Come abbiamo affermato prima, nella parte storica, il processo matrimoniale ogni tanto viene modificato, rispondendo alle esigenze temporanee. Già

20 Cf. Paulus VI, Litterae apostolicae motu proprio datae quibus normae quaedam statuunturad processus matrimonialis expeditius absolvendos (28.03.1971), AAS 63 (1971), p. 441-446.

21 Pontificium Consilium de Legum Textibus, Instructio, 25 ian.2005, Romae, ex. Aed. Consilii, Instructio servanda a tribunalibus dioecesanis et interdiocesanis in pertractandis causis nullitatis matrimonii, Civitas Vaticana 2005.

Cf. M. J. Arroba Conde, Diritto processuale canonico, Roma 2012, p. 613-625.

23 Cf. Can. $1682 \S 1$ del Codice di Diritto Canonico (dopo: CIC).

24 Cf. M. del Pozzo, op. cit., p. 19. 
dalla codificazione del 1983 si sentiva i voci di fare alcune modificazioni ${ }^{25}$. Senza dubbi possiamo dire, che il Papa Francesco con il suo contributo porta in diritto canonico un nuovo approccio ${ }^{26}$. Dobbiamo sottolineare ancora, che il Papa Francesco ci spiega il valore giuridico della nuova legislazione dicendo così: ,le leggi di riforma del processo matrimoniale susciate abrogano e derogano ogni legge o norma contraria vigente"27. I principi della riforma del Papa Francesco:

a) Il principio dell'indissolubilità - il Romano Pontefice afferma chiaramente, che come principio cardine di tutta la riforma rimane la difesa dell'indissolubilità del matrimonio ${ }^{28}$. Dice Papa Bergoglio: „ho costituito un gruppo di persone ... abbozzassero un progetto di riforma, fermo restando comunque il principio dell'indissolubilità del vincolo matrimoniale"29. Il Supremo Legislastore ha scelto per le cause di nullità del matrimonio la via giudiziale, che deve garantire l'indossulubilità del matrimonio matrimonio cristiano come sacramento è indissolubile. Secondo Papa Francesco l'altra modalità, di svolgere i processi matrimoniali per la via amministrattiva apre la possibilità di pensare al divorzio cattolico ${ }^{30}$.

25 GiànelSinodo sull'Eucaristia i padrisinodalinella Proposito n. 40 hanno richiesto: „Nello stesso tempo il sinodo auspica che sia fatto ogni possibile sforzo sia per assicurare il carattere pastorale, la presenza e la corretta e sollecita attività dei tribunali ecclesiastici per le cause di nullità matrimoniale, sia per aprofondire ulteriormente gli elementi essenziali per la validità del matrimonio, anche tenedo conto dei problemi emergenti dal contesto di profonda trasformazione antropologica del nostro tempo, dal quale gli stessi fedeli rischiano di esser condizionati specialmete in mancanza di una solida formazione cristiana"; Practicamente lo stesso si poteva sentire dai rappresentanti della dottrina - ad esempio il prof. Mons. A. Stankiewicz, già decano della Rota Romana, proponeva qualche modo di celerità dei processi matrimoniali; Vedi: A. Stankiewicz, La celerità nelle cause di nullità matrimoniale: aspetti operative, [in:] Ius et Matrimonium, Temi di diritto matrimoniale e processuale canonico, H. Franceschi, M. A. Ortiz (a cura di), Roma, 2015, p. 217-236.

26 Cf. V. Andriano, La normativa canonica sul matrimonio e la riforma del processo di nullità, Città del Vaticano 2016, p. 169.

27 Francesco, Rescritto ex Audientia SS.mi” sulla nuova legge del procesco matromoniale, [in:] Tribunale Apostolico della Rota Romana, Sussio applicativo del Motu pr. Mitis Iudex Dominus Iesus, Città del Vaticano 2016, p. 49.

28 Il Codice di diritto Canonico nel canone 1056 mostra due proprietà essenziali del matrimonio: l'unità e proprio l'indissolubilità.

29 Cf. MIDI, p. 2.

30 Cf. Francesco, Conferenza stampa durante il volo di ritorno dagli Stati Uniti d'America, 27.IX.2015. 
b) Il principio della celerità - la velocizzazione è infatti lo scopo più facilmente percepibile e desiderabile da parte dei fedeli. La più attesa è condivisa misura di riordino non a caso è stata proprio l'abolizione della doppia decisione conforme. Poi anche nel processus brevior si vede che si risparmia tempo, perché la velocizzazione si esprime nell'unicità della sessione istruttoria o nell'elleminazione di altre formalità pre-decisorie (per esempio: conclusione della causa, pubblicazione degli atti e discussione). Il Papa spiega chiaramente, che nella riforma non favorisce la nullità dei matrimoni, ma la celerità dei processi ${ }^{31}$.

c) Il principio della semplicità - iusta simplicitatis - indica che la promozione della snellezza non può tradursi nella compressione delle garanzie essenziali e del diritto di difesa. Afferma Papa Francesco: „ho deciso di dare con questo Motu proprio disposizioni con le quali si favorisca non la nullità dei matrimoni, ma la celerità dei processi, non meno che una giusta semplicità ${ }^{32}$.

d) Il principio della prossimità o vicinanza - interessa sopratutto la fruibilità e accessibilità del giudice. Il principio funge quasi da perno della conversione delle strutture ecclesiastiche, ha determinato una svolta dell'organizzazione giudiziaria ecclesiastica $^{33}$. Il Decano della Rota Romana e Presidente della Pontificia Commissione per la riforma del processo matrimoniale, Mons. P. Pinto proponeva un esempio significativo: „In alcune circostanze particolari il Vescovo, come pastore e giudice del suo gregge, potrebbe consegnare personalmente la sentenza di nullità alle parti interessate. Sarebbe un segno di prossimità evangelica ai fedeli, in molti casi feriti da anni di sofferenza ${ }^{34}$.

e) Il principio dell'economicità - questo principio trova un'espressa formulazione nel VI Criterio del MIDI: „Insieme con la prossimità del giudice curino per quanto possibile le Conferenze Episcopali, salva la giusta e dignitosa retribuzione degli operatori dei tribunali, che venga assicurata la gratuità delle procedure, perché la Chiesa, mostrandosi ai fedeli madre generosa, in una materia così strettamente legata alla salvezza delle anime manifesti l'amore gratuito di Cristo dal quale tutti siamo salvati ${ }^{35}$.

Cf. MIDI, p. 2-3.

32 Ibidem, p. 3.

Cf. M. del Pozzo, op. cit., p. 32-33. nuovo processo matrimoniale, "L'Osservatore Romano", 7.X.2015., p. 6-7. 
Questo principio viene molto spesso indicato nell'insegnamento del Papa Francesco - è una aspirazione della gratuità dell'intera disciplina sacramentale ${ }^{36}$.

\section{I compiti processuali del Vescovo diocesano secondo la riforma francescana}

Leggendo il Motu proprio Mitis Iudex Dominus Iesus si nota subito, che i primi destinatari di questo documento sono vescovi. In verità, Papa Francesco pone al centro della sua riforma la figura del Vescovo diocesano ${ }^{37}$. Il Concilio Vaticano II ci insegna, che i vescovi sono per la divina istituzione succeduti al posto degli Apostoli ${ }^{38}$. Nella consecrazione episcopale ricevono la pienezza del sacramento d'ordine, ed esercitano l'ufficio di santificare, di insegnare e di governare ${ }^{39}$. I vescovi reggono le Chiese particolari a loro affidate come vicari e legati di Cristo. In virtù di questa potestà i vescovi hanno il sacro diritto e davanti al Signore il dovere di dare leggi ai loro sudditi, di giudicare e di regolare tutto quanto appartiene al culto e all'apostolato ${ }^{40}$. Come la conseguenza, il Codice di Diritto Canonico dal 1983 (lo chiamiamo spesso come l'ultimo documento del Vaticanum II) afferma che il Vescovo diocesano in ogni Chiesa locale è giudice di prima istanza, può esercitare questa potestà personalmente o tramite altri ${ }^{41}$. Già nell'allocuzione alla Segnatura Apostolica dell' 8 novembre 2013, il Papa Francesco aveva posto l'accento sulla responsabilità episcopale nell'amministrazione della giustizia: „La vostra attività è volta a favorire l'opera dei tribunali ecclesiasctici, chiamati a rispondere adeguatamente ai fedeli che si rivolgono alla giustizia della Chiesa per ottenere una giusta decisione. Vi adoperate perché funzionino bene, e sostenete la responsabilità dei vescovi nel formare idonei ministri della giustizia"42. Secondo questi pensieri di Papa Francesco nella nuova legislazione si afferma che lo

$36 \quad$ Cf. M. del Pozzo, op. cit., p. 34-35.

37 Sotto il termine vescovo diocesano dobbiamo capire anche altri capi delle Chiese particolari secondo la norma del can. 368; ovviamente quelli privilegi non vengono affidati ai vescovi ausiliari.

38 Concilio Vaticano II, Costituzione dogmatica sulla Chiesa Lumen Gentium, [in:] I documenti del Concilio Vaticano II, Milano 2012, 20.

39 Ibidem, 21.

$40 \quad$ Ibidem, 27.

$41 \quad$ Cf. CIC $1419 \S 1$.

42 Francesco, Allocuzione Questa vostra sessione alla sesione plenaria del Supremo tribunale della Segnatura apostolica, 8 novembre, AAS 105 (2013), p. 1152. 
stesso Vescovo è giudice. Nel III criterio del MIDI, basando sull'insegnamento conciliare invita i vescovi ad mettere in pratica il loro compito di essere non solo capo e pastore, ma anche il giudice tra i fedeli a loro affidati ${ }^{43}$. Annalizzando la nuova normativa possiamo indicare i compiti processuali del Vescovo diocesano:

a) Soltanto il Vescovo diocesano ha il diritto di costituire il giudice unico (sempre deve essere un chierico) nel processo matrimonia$\mathrm{le}^{44}$. Si può fare così, soltanto sotto alcuni condizooni: l'impossibilità di costituire il tribunale collegiale e devono essere nominati due assessori. ${ }^{45}$ Alcuni canonisti ci ricordano, che non si dovrebbero costituire un giudice monocratico per la mancanza di mezzi finanziari (per la volontà di compiere il principio di graduità) ${ }^{46}$.

b) Il Vescovo diocesano come unico giudice nel processus brevior (ipsi episcopo dioecesano competit - giudicare in processus brevior è la competenza che tiene soltanto il Vescovo diocesano $\left.{ }^{47}\right)$ - con la presente legislazione il Romano Pontefice crea una grande novità: il processus brevior (il processo più breve). Secondo il canonista M. del Pozzo questo processo costituisce la più sorprendente innovazione del procedimento di accertamento della nullità del matrimonio voluta da Papa Francesco ${ }^{48}$. Al questo processo si rivolgono i canoni 1683-1687. Come spiega Papa Francesco il processus brevior è una nuova forma di processo matrimoniale, da applicarsi nei casi in cui l'accusata nullità del matrimonio è sostenuta da argomenti particolarmente evidenti ${ }^{49}$. In quel processo viene mostrato come il maggiore garante dell'unità cattolica nella fede e nella disciplina. Dobbiamo sottolineare, che il Vescovo in quel processo, se ha raggiunto la certezza morale sulla nullità del matrimonio, deve pronunziare la sentenza. Se non raggiunge quella certezza morale, la causa torna al processo

Cf. MIDI, p. 3 .

$44 \quad$ Cf. Ibidem.

$45 \quad$ Cf. CIC $1673 \S 4$.

46 Cf. M. del Pozzo, op. cit., p. 35.

$47 \quad$ Cf. CIC 1683.

48 Cf. M. del Pozzo, op. cit., p. 11. Lo stesso canonista ci da una delle migliori spiegazioni come dovremmo capire quello processo: „Nuova è sicuramente la forma e la procedura, non certo il principio e la competenza”, in M. del Pozzo, op. cit., p. 41-42. 
ordinario $^{50}$. Questo significa, che il Vescovo può pronunziare soltanto la sentenza positiva - pro nullitate.

c) Il Vescovo diocesano deve essere un segno della conversione delle strutture ecclesiastiche, ciò practicamente significa, che il Vescovo è responsabile per il funzionamento del suo Tribunale ecclesiastico nel prima istanza ${ }^{51}$.

d) Il Vescovo diocesano è il giudice di prima istanza nelle cause di nullità del matrimonio nella sua diocesi, può esercitare questa potestà giudiziale personalmente o per mezzo di altri ${ }^{52}$.

e) Il Vescovo diocesano ha il diritto di costituire per la sua diocesi il tribunale diocesano della prima istanza per le cause di nullità del matrimonio. Ovviamente, il Supremo Legislatore salva la facoltà allo stesso Vescovo di accedere a un altro tribunale diocesano o interdiocesano ${ }^{53}$.

f) Il Vescovo diocesano deve presentare suo voto nella causa per la dispensa super rato ${ }^{54}$.

g) Il Vescovo come ordinario del luogo ${ }^{55}$ può emanare il divieto di contrare delle nuove nozze, dopo che la sentenza ha dichiarato la nullità del matrimonio è divenuta esecutiva ${ }^{56}$.

h) Il Vescovo diocesano, come ordinario del luogo deve provvedere affinché al più presto si faccia menzione nei registri dei matrimoni e dei battezzati della nullità di matrimonio decretata e degli evantuali divieti stabiliti ${ }^{57}$.

Cf. CIC 1687 § 1.

Cf. MIDI, p. 3-4.

Cf. CIC 1673 § 1. La normativa precedente non parlava del Vescovo come giudice.

Cf. CIC 1673 § 2. La normativa di quel canone si riguarda molto verso i tribunali italiani. In Italia, secondo la volontà del Papa Pio XI con il Motu proprio Qua cura lascia la competenza di giudicare nelle cause di nullità del matrimonio ai Tribunali Regionali. Fin'ora i Tribunal Regionali: Piemontese, Lombardo e Veneto seguono la strada vecchia. Solo i Vescovi della Conferenza Episcopale Siciliana hanno modificato le competenze dei tribunali, creando i tribunali diocesani e interdiocesani, nel posto del Tribunale Regionale Siciliano.

Cf. CIC $1678 \S 4$. Si sembra che rimane in vigore la legislazione dall'Istruzione Dignitas connubii art. $154 \S 3$ : ,Quanto al voto del Vescovo, nulla vieta che esso sia riedatto in calce al voto dello stesso tribunale, mediante sottoscrizione di quest'ultimo, con l'assicurazione della giusta e proporzionata causa per la concessione della dispensa e l'assenza di scandalo da parte dei fedeli'.

Cf. CIC 134.

Cf. Ibidem, 1682 § 1.

$57 \quad$ Cf. Ibidem, 1682 § 2. 
i) Il Vescovo diocesano come suffraganeo può ammetere l'appello dal sentenza del processus brevior emessa dal Metropolita. Il legislatore dice, che quel diritto ha il suffraganeo più anziano ${ }^{58}$. Secondo la risposta del Pontificio Consiglio per i Testi Legislativi sotto il termine suffraganeo più anziano si capisce il Vescovo della sedia vescovilie più anziana nella metropolia. ${ }^{59}$ Questo significa, che alcuni vescovi (in specifico i metropoliti e il suffraganeo più anziano di ciascuna provincia) avranno non solo l'onore di giudicare in prima istanza i processi più brevi delle loro diocesi, ma dovranno anche intervenire in secondo grado per i casi di apello contro la sentenza di primo grado emessa da un vescovo ${ }^{60}$.

j) Il Vescovo diocesano può dichiarare la nullità del matrimonio nel processo documentale (cann. 1688-1690 ${ }^{61}$, cioè nel processo dove la nullità può derivare dagli impedimenti o dal difetto di forma. Si può dichiarare la nullità del matrimonio da un documento ${ }^{62}$.

k) Nella terza parte del Motu proprio Mitis Iudex Dominus Iesus nelle Regole procedurali per la trattazione delle cause di nullità matrimoniale troviamo ancora altri compiti dei Vescovi, che si sembrano più pastorali:

- Il Vescovo diocesano, assieme con parroco condivide la sollecitudine pastorale per i fedeli che sono in difficoltà, sopratutto i coniugi separati e divorziati ${ }^{63}$.

- Il Vescovo diocesano può stabilire (al livello diocesano o interdiocesano) una struttura stabile con lo scopo pastorale di consulenza per preparae meglio il svolgimento dell'indagine. Si consiglia anche di redigere un Vademecum, che riporta gli elementi essenziali per il più adeguato svolgimento dell'indagine $^{64}$. Questi strumenti possono servire per eventuale Cf. Ibidem, 1687 § 3.

Cf. Pontificio Consiglio per i Testi Legislativi, Circa il suffraganeus antiguior nel nuovo can. 1687 \& 3 Mitis Iudex, Vaticano 13 ottobre 2015, http:// http:// www.delegumtextibus.va/content/dam/testilegislativi/risposte-particolari/ Procedure\%20per\%20la\%20Dichiarazione\%20della\%20Nullit\%C3\%A0\%20 matrimoniale/Circa\%20i1\%20suffraganeus\%20antiquior\%20nel\%20nuovo\%20 can.\%201687\%20\%C2\%A73\%20Mitis\%20Iudex.pdf (4.11.2016).

Cf. M. Mingardi, Il ruolo del vescovo diocesano, [in:] Redazione di Quaderni di diritto ecclesiale (a cura di), La riforma dei processi matrimoniali di Papa Francesco, Milano 2016, p. 104-105.

Cf. CIC 1688.

Cf. M.J. Arroba Conde, op. cit., p. 626-628.

Cf. MIDI, p. 14.

64 Cf. Ibidem, p. 14-15. 
introduzione della causa da parte dei coniugi o del loro patrono davanti al tribunale competente ${ }^{65}$. Questo cammino di accompagnamento può aiutare a superare in maniera soddisfacente le crisi matrimoniali, ma è anche chiamato a verificare nei casi concreti, la verifica della validità o meno del matrimonio è raccogliere elementi utili per l'eventuale celebrazione del processo giudiziale: ordinario o breviore ${ }^{66}$.

Parlando dei compiti dei Vescovi diocesani nel processo matrimoniale secondo la riforma del Papa Francesco dobbiamo dire anche un'po sui compiti processuali del Metropolita ${ }^{67}$. Il suo compito fondametale è vigilare la fede e disciplina ecclesiastica nella sua provincia ecclesiastica $^{68}$. Si sembra, che il Metropolita dobrebbe essere un segno della giusta interpretazione e introduzione della riforma del Papa Francesco nella sua provincia ecclesiastica. Fuorchè dei suoi propri compiti come arcivescovo diocesano, il Papa Francesco prevede l'appello alla Sede Metropolitana. Nel Criterio V del Motu proprio Francesco dice: „Convienie che si ripristini l'appello alla Sede del Metroplita, giacchè tale ufficio di capo della provincia ecclesiastica, stabile nei secoli, è un segno distintivo della sinodalità nella Chiesa"69. Poi nel processus brevior il Legislatore ha previsto l'appello verso il Metropolita ${ }^{70}$, si sembra, che è uguale nel processo documentale, dove l'appello dovrebbe andare verso il Metropolita ${ }^{71}$.

Alcuni compiti il Papa Francesco ha previsto anche verso la Conferenza dei Vescovi. Ovviamente, ogni vescovo diocesano è un membro ordinario di una Conferenza Episcopale. Nel Criterio VI Papa Francesco scrive, che Le Conferenze dei Vescovi devono essere spinte dall'ansia apostolica di raggiungere i fedeli dispersi, devono rispettare il proprio diritto dei Vescovi di organizzare la potestà giudiziale nella propria Chiesa locale. Il compito principale che ricevono le Conferenze Episcopali devono curare la giusta dignitosa retribuzione degli

\footnotetext{
65 Cf. Ibidem, p. 15.

66 Cf. Tribunale Apostolico della Rota Romana, Sussidio applicativo del Motu pr. Mitis Iudex Dominus Iesus, Città del Vaticano 2016, p. 14.

67 Il canone 435 spiega, che il Metropolita è l'arcivescovo, che presiede di una provincia eccelesiastica.

$68 \quad$ Cf. CIC $436 \S 1$.

69 MIDI, p. 4.

$70 \quad$ Cf. CIC 1687 § 3.

${ }^{71}$ Cf. Ibidem, 1688-1689.
}

\section{Canon law}


operatori dei tribunali, ed anche devono sistemare la gratuità dei processi ${ }^{72}$. Ci sembra, che il Papa Francesco vuole, che per la stessa Conferenza dei Vescovi esisteranno le stesse regole. Ha espresso questo pensiero anche nella ultima esortazione Amoris laetitia, (che è stata emanata dopo Mitis Iudex Dominus Iesus, ma rappresenta lo stesso pensiero teologico; leggendo Amoris laetitia capiamo meglio le idee della riforma del processo matrimoniale). Francesco scrive così: „Naturalmente, nella Chiesa è neccessaria una unità di dottrina e di prassi, ma ciò non impedisce che esistano diversi modi di interpretare alcuni aspetti della dottrina o alcune conseguenze che da essi derivano"73.

\section{Conclusione}

La riflessione sulla riforma del processo matrimoniale ci ha fatto vedere, che il Supremo Legislatore Papa Francesco ha voluto mettere al centro della riforma la figura del Vescovo diocesano ${ }^{74}$. Il Motu proprio Mitis Iudex Dominus Iesus pone, che proprio davanti al Vescovo diocesano si crea un nuovo, particolare e personale campo della responsabilità. Come avevamo detto, il Vescovo adesso non è soltanto responsabile per costituire e vigilare le strutture giudiziare nella sua Chiesa locale, ma è chiamato anche ad partecipare e compiere personalmente il compito del primo giudice del suo gregge. La nuova normativa prevvede tanti procedimenti in cui la presenza del Vescovo diocesano è indispensabile (sopratutto processus brevior). Adesso si vede la più forte coinvolgimento del Vescovo nel processo ordinario (costituire giudice unico, nominare i giudici laici) nel processus brevior ed anche nel processo documentale (può emanare la sentenza). Il Vescovo diocesano ha ricevuto anche il compito di essere sede di'apello per le sentenze del suo Metropolita. Annalizando la nuova leggislazione e la posizione del Vescovo diocesano possiamo scoprire, che il Papa sta facendo una grande riforma delle strutture ecclesiastiche, tornando ai radici pastorali, e vuole mettere in pratica l'insegnamento del Concilio Vaticano II. Come abbiamo indicato, il Papa Francesco sequendo le orme dei suoi Predecessori, svolge l'opera,che viene fatta nella continuità storica. ${ }^{75}$ Secondo i padri conciliari e secondo Papa Francesco il Vescovo diocesano non viene percepito soltanto come Pastor bonus,

\footnotetext{
72 Cf. MIDI, p. 4-5.

73 Francesco, Esortazione apostolica sull'amore nella famiglia Amoris laetitia, Città del Vaticano 2016, 3.

74 Cf. Tribunale Apostolico della Rota Romana, op. cit., p. 9-10.

75 Cf. M. del Pozzo, op.cit., p. 41-42.
} 
ma anche come iustus iudex ${ }^{76}$. Lo scopo principale della riforma rimane la salvezza delle anime ${ }^{77}$, ma la nuova legislazione contribuisce anche un grande rinnovanento e una nuova sfida nel svolgimento del ministero episcopale.

\section{OSOBA I ZADANIA PROCESOWE BISKUPA DIECEZJALNEGO W PROCESIE MAŁŻEŃSKIM W ŚWIETLE REFORMY PAPIEŻA FRANCISZKA}

Od początku pontyfikatu papieża Franciszka słyszymy w jego przepowiadaniu nawoływanie do reformy struktur kościelnych. Swoją pastoralno-ewangeliczną koncepcję funkcjonowania Kościoła Bergoglio przedstawił w adhortacji Evangelii Gaudium. Naturalnie, reforma dokonywana przez Biskupa Rzymu dotyczy również prawa kanonicznego. Kierowany głosami hierarchów zgromadzonych na Synodzie Biskupów w 2014 roku oraz dobrem duchowym wiernych, którzy oczekują na wyjaśnienie swojej sytuacji kanonicznej, papież Franciszek 8 września 2015 roku promulgował Motu proprio Mitis ludex Dominus lesus tak, by przyspieszyć i uprościć proces stwierdzenia nieważności małżeństwa. Wolą Papieża było ty, by serca wiernych nie były zbyt długo zniewolone przez mroki wątpliwości z powodu opóźnień w wydaniu wyroku. W centrum swojej reformy Ojciec Święty postawił postać biskupa diecezjalnego, który wraz z Biskupem Rzymu staje się gwarantem katolickiej jedności w wierze i dyscyplinie. Według założeń reformy, biskup nie tylko organizuje i odpowiada za strukturę sądowniczą w swoim Kościele lokalnym, ale ma również wypełniać osobiście zadanie pierwszego sędziego wiernych, dla których został ustanowiony pasterzem. Nowe ustawodawstwo daje biskupowi możliwość ustanowienia sędziego jednoosobowego oraz wprowadza instytucję processus brevior, który przewidziany jest w sytuacjach szczególnie oczywistych. Jedynym sędzią w tym procesie jest sam biskup. Wydaję, że reforma procesu małżeńskiego, dokonana w duchu ciągłości historycznej, przyniesie wiele owoców duchowych i przyczyni się do postrzegania osoby biskupa diecezjalnego nie tylko jako pastor bonus, ale jednocześnie jako iustus iudex.

Słowa kluczowe: Franciszek, proces małżeński, biskup diecezjalny, sędzia, nieważność małżeństwa, processus brevior, reforma.

76 Cf. T. Rozkrut, Odpowiedzialnośćbiskupa diecezjalnego, metropolity oraz konferencji biskupów za wspótczesny proces matżeński, [in:] Proces matżeński wedtug motu proprio Mitis Iudex Dominus Iesus, J. Krajczyński (a cura di), Płock 2015, p. 47.

77 Cf. CIC 1752; MIDI, p. 1-3. 


\section{Bibliografia:}

1. Acte et documente Consilio Oecumenico Vaticano II apparando, series I, Appendicis vol. II, p. II, Romae 1961.

2. Andriano V., La normativa canonica sul matrimonio e la riforma del processo di nullità, Città del Vaticano 2016.

3. Arroba Conde M.J., Diritto processuale canonico, Roma 2012.

4. III Asemblea Generale Straordinaria del Sinodo dei Vescovi, Relatio Synodi, Città del Vaticano 2014.

5. Benedictus XIV, Constitutio Dei miseratione, [in:] Codicis Canonicifontes, I, P. Gasparri (a cura di), Romae 1923.

6. Concilio Vaticano II, Costituzione dogmatica sulla Chiesa Lumen Gentium, [in:] I documenti del Concilio Vaticano II, Milano 2012.

Canon law

7. Fantappiè C., Storia del diritto canonico e delle istituzioni della Chiesa, Bologna 2011.

8. Francesco, Allocuzione Questa vostra sessione alla sesione plenaria del Supremo tribunale della Segnatura Apostolica, 8 novembre, AAS 105 (2013).

9. Francesco, Conferenza stampa durante il volo di ritorno dagli Stati Uniti d'America, 27.IX.2015.

10. Francesco, Esortazione apostolica sull'amore nella famiglia Amoris laetitia, Città del Vaticano 2016.

11. Francesco, Rescritto ex Audientia SS.mi" sulla nuova legge del procesco matromoniale, [in:] Tribunale Apostolico della Rota Romana, Sussio applicativo del Motu pr. Mitis Iudex Dominus Iesus, Città del Vaticano 2016.

12. Francesco, Udienza ai rappresentati dei media, Discorso del Santo Padre, Aula Paolo VI, 16 marzo 2013, http://w2.vatican.va/content/francesco/it/ speeches/2013/march/documents/papa-francesco_20130316_rappresentanti-media.html. (10.01.2017).

13. Góralski W., Wprowadzenie do motu proprio Mitis Iudex Dominus Iesus, [in:] Proces matżeński wedtug motu proprio Mitis Iudex Dominus Iesus, J. Krajczyński (a cura di), Płock 2015.

14. Ioannes Paulus Pp. II, Codex Iuris Canonici, AAS LXXV Pars II (1983).

15. Lettera Apostolica Mitis Iudex Dominus Iesus data Motu Proprio dal Santo Padre Francesco sulla riforma del processo canononico per le cause di dichiarazione di nullità del matrimonio nel Codice di Diritto Canonico (dopo: MIDI), Coletti a San Pietro 2015.

16. Mingardi M., Il ruolo del vescovo diocesano, [in:] La riforma dei processi matrimoniali di Papa Francesco, Redazione di Quaderni di diritto ecclesiale (a cura di), Milano 2016.

17. Paulus VI, Litterae apostolicae motu proprio datae quibus normae quaedam statuuntur ad processus matrimonialis expeditius absolvendos (28.03.1971), AAS 63 (1971).

18. Pinto P., Speranza e non paure, Intervista al Decano della Rota romana sul nuovo processo matrimoniale, "L'Osservatore Romano", 7.X.2015.

19. Pius Pp. X, Constitutio apostolica Sapienti Consilio, Romae 29.06.1908, http:// https://w2.vatican.va/content/pius-x/la/apost_constitutions/documents/hf_p-x_apc_19080629_sapienti-consilio-index.html (4.11.2016). 
20. Pontificio Consiglio per i Testi Legislativi, Circa il suffraganeus antiquior nel nuovo can. 1687 \& 3 Mitis Iudex, Vaticano 13 ottobre 2015, http://www. delegumtextibus.va/content/dam/testilegislativi/risposte-particolari/Procedure\%20per\%20la\%20Dichiarazione\%20della\%20Nullit\%C3\%A0\%20 matrimoniale/Circa\%20il\%20suffraganeus\%20antiquior\%20nel\%20 nuovo\%20can.\%201687\%20\%C2\%A73\%20Mitis\%20Iudex.pdf (4.11.2016).

21. Pontificium Consilium de Legum Textibus, Instructio, 25 ian.2005, Romae, ex. Aed. Consilii, Instructio servanda a tribunalibus dioecesanis et interdiocesanis in pertractandis causis nullitatis matrimonii, Civitas Vaticana 2005.

22. Pozzo del M., Il processo matrimoniale più breve davanti al Vescovo, Roma 2016.

23. Rozkrut T., Odpowiedzialność biskupa diecezjalnego, metropolity oraz konferencji biskupów za współczesny proces matżeński,[in:] Proces matżeński wedtug motu proprio Mitis Iudex Dominus Iesus, J. Krajczyński (a cura di), Płock 2015.

24. Stankiewicz A., La celerità nelle cause di nullitá matrimoniale: aspetti operative, [in:] Ius et Matrimonium, Temi di diritto matrimoniale e processuale canonico, H. Franceschi, M. A. Ortiz (a cura di), Roma, 2015.

25. Tribunale Apostolico della Rota Romana, Sussidio applicativo del Motu pr. Mitis Iudex Dominus Iesus, Città del Vaticano 2016. 